\title{
La vie avant la guerre au monastère de Sainte-Thècle en Syrie
}

Anna Poujeau

Cet article décrit une réalité passée. La guerre en Syrie a quasiment tout détruit et n'a épargné personne. En 2013, au début du mois de décembre, les moniales de la communauté monastique de Sainte-Thècle ont été contraintes d'abandonner leur monastère.

Jusqu'à ce départ forcé, comme les autres moniales, hajja Tedocia, dont il sera plus particulièrement question dans cet article, ne pouvait imaginer qu'elle devrait un jour vivre loin de Sainte-Thècle. Celle qui avait été la plus jeune moniale de Syrie et qui était désormais la doyenne du couvent n'avait pas d'autre choix que de le quitter.

Les obus, les tirs d'artillerie et les roquettes avaient éventré ses épaisses murailles, pulvérisé ses lourdes portes en bois massif et fait s'effondrer la coupole de l'église. L'harmonieuse cour centrale avec son bassin au milieu n'était plus qu'un tas de gravats, la vaste terrasse surplombant la vallée où les moniales aimaient se promener tranquillement le soir venu n'offrait désormais plus aucun repos.

Le monastère, lieu des derniers espoirs de millions de Syriens, n'en était plus que le tombeau ${ }^{1}$.

\section{RELATION INTIME AU DIVIN}

L'aube point à peine et une froide journée d'hiver s'annonce lorsque hajja ${ }^{2}$ Marina ouvre les portes d'entrée du monastère grec orthodoxe de Sainte-Thècle ${ }^{3}$. L'imposante bâtisse construite à flanc d'une falaise de la région montagneuse du Haut Qalamûn ${ }^{4}$, au nord de Damas, domine le célèbre petit village chrétien de Ma ${ }^{\complement}$ lûlâ. Parce qu'il abrite le tombeau de sainte Thècle ${ }^{5}$, qui selon la légende locale y aurait trouvé refuge et y aurait été ensevelie en

\footnotetext{
${ }^{1}$ J'ai consacré à l'abandon forcé du monastère par les moniales une communication, « Les moniales de SainteThècle et les combattants du Front al-Nosra : un "enlèvement" et une libération problématiques » lors de la journée d'étude Les Processus d'assignation politico-religieux dans le conflit syrien du 15 décembre 2016 à Paris ainsi qu'un article à paraître au sujet de l'affaire retentissante du départ des moniales de Sainte-Thècle pour Yabroud, ville alors sous contrôle de la rébellion.

${ }^{2}$ Hajja vient du terme hajj qui désigne chez les chrétiens le pèlerinage à Jérusalem. Cela fait référence au fait que le monastère se trouve sur la route du pèlerinage à Jérusalem et en constituait dans le passé une étape. Cela n'est plus d'actualité depuis la fermeture des frontières entre la Syrie et Israël.

${ }^{3}$ Cet article se fonde sur plusieurs séjours de terrain effectué au monastère entre 2003 et 2007 et une monographie Poujeau Des monastères en partage. Sainteté et pouvoir chez les chrétiens de Syrie, Société d'ethnologie, Nanterre, 2014.

${ }^{4}$ La bâtisse monastique a été construite à partir du XVII siècle par l'Église grecque orthodoxe d'Antioche et de tout l'Orient. Néanmoins, on retrouve une trace bien plus ancienne de la présence de moines ermites qui vivaient dans les grottes autour de celle abritant le tombeau de la sainte (Maraval, 1985 référence à compléter : p,86). ${ }^{5}$ Sainte Thècle est l'une des premières saintes du christianisme et son hagiographie est bien connue. Fille du gouverneur de Konya en Turquie, elle fut convertie au christianisme par saint Paul de passage dans la ville.
} 
45 après J.-C., le monastère est un lieu de pèlerinage important pour des milliers de dévots chrétiens et musulmans ${ }^{6}$.

Une quinzaine de religieuses - postulantes, novices et moniales - lui consacrent leur existence et se tiennent prêtes à accueillir les visiteurs qui se rendent sur son tombeau pour lui adresser des prières ou encore, la remercier pour des grâces obtenues. Leur quotidien, rythmé avec régularité, commence par les prières de l'orthros (du grec : «aurore »). Ainsi après s'être assurée qu'aucun visiteur n'attende aux portes du monastère, hajja Marina monte les trois grands escaliers de pierre qui débouchent sur la cour carrée et l'église. Une autre volée de marches plus étroites permet d'accéder sur deux étages aux petites cellules monastiques alignées le long de balcons filants.

Au moment où hajja Marina traverse la cour carrée, elle croise d'autres moniales qui, comme elle, se rendent à l'église. Dans celle-ci encore faiblement éclairée par la lumière des premières chandelles, résonnent les premières prières de la journée.

Hajja Tedocia, la plus âgée des moniales, est la dernière à quitter sa cellule. Petite, les yeux pétillants et le visage barré d'un sourire qui ne paraît jamais la quitter, elle porte l'habit monastique depuis presque soixante ans. Sans hâte, elle accomplit soigneusement tous les gestes rituels. Au seuil de l'église, elle s'arrête quelques instants pour embrasser la porte ornée de plaque de cuivre martelé avant d'y poser son front en signe de dévotion. Une fois le pas de la porte franchi, elle se dirige vers l'icône posée sur le pupitre à gauche de l'entrée. Elle fait un signe de croix, le premier d'une longue série qui ponctue quasiment tous ses actes quotidiens. C'est une petite métanie ${ }^{7}$ : hajja Tedocia se baisse vers le sol et le touche de trois doigts de la main droite, pouce, index et majeur réunis qui symbolisent la Trinité. Puis elle se redresse lentement et fait le signe de croix. Elle accomplit cette petite métanie trois fois de suite tout en murmurant quelques suppliques. Elle se penche ensuite vers l'icône afin de l'embrasser trois fois. Entre chaque baiser, elle pose quelques instants son front sur l'icône en fermant les yeux. Enfin, de sa main droite, elle saisit deux ou trois cierges déposés à côté et trace un signe de croix sur l'icône. Elle se place ensuite devant le brûloir situé à proximité de

\footnotetext{
Soumise à différents supplices par ses parents païens, la légende plus largement répandue dans le monde, situe son tombeau à Séleucie. C'est d'ailleurs un lieu de pèlerinage très fréquenté.

${ }^{6}$ La sainte, comme tous les autres saints de la région, délivre ses miracles aux pèlerins à la fois chrétiens et musulmans. Ainsi, dans la région, de nombreux musulmans visitent les lieux saints chrétiens dans l'espoir de voir leurs vœux de guérison, fertilité, etc. exaucés.

${ }^{7}$ Le terme « métanie » (du grec metanoïa, « repentir, pénitence, conversion »), désigne deux postures corporelles précises : une grande inclination du buste vers le sol accompagnée d'un geste de la main droite qui va toucher le sol avant de se signer en se relevant (petite métanie) et une prosternation de tout le corps allongé à terre (grande métanie). Entre deux grandes métanies, les religieuses se signent.
} 
l'icône où un peu plus tôt, d'autres cierges ont été déposés par les religieuses. Hajja Tedocia allume les cierges l'un après l'autre puis les éteint en soufflant rapidement dessus. Elle renouvelle son geste deux ou trois fois pour chaque cierge avant de les fixer dans le sable du brûloir où ils se consumeront pendant quelques heures ${ }^{8}$.

Tandis que les autres religieuses chantent les psaumes du jour, elle traverse d'un pas lent le narthex et la nef, passe sous la coupole et le polyeleos ${ }^{9}$ et s'arrête devant l'iconostase. Elle accomplit à nouveau une petite métanie devant chacune des quatre grandes icônes qui le composent puis les embrasse. Lorsque hajja Tedocia atteint l'extrémité droite de l'iconostase, elle franchit deux marches pour passer du côté de la sacristie. En effet, son statut de femme ménopausée l'autorise à se tenir aux côtés du pope et à l'assister durant les liturgies ${ }^{10}$. Ce dernier qui habite au village à quelques mètres du monastère ne se présente à l'église qu'une heure environ après le début des psalmodies ${ }^{11}$.

Dans le sanctuaire, hajja Tedocia fait brûler quelques minutes de l'encens dans un encensoir à main. Puis, depuis le seuil de l'iconostase, elle le tend à Belagia, la supérieure de la communauté, qui elle-même ne peut pas pénétrer dans le sanctuaire. Celle-ci va déambuler plusieurs minutes dans l'église pour y diffuser l'encens mais avant cela, elle s'arrête avec l'encens fumant devant chacune des moniales, qui tour à tour, s'incline et se signe tout en continuant de psalmodier. Belagia se dirige ensuite vers le fond du narthex tout en s'arrêtant devant chacune des icônes pour y faire un signe de croix avec l'encensoir.

Puis elle retourne vers l'iconostase en marquant quelques instants d'arrêt devant les rares fidèles, deux ou trois, assis sur les bancs de l'allée gauche du narthex. Ce sont les visiteurs ayant passé la nuit au monastère ou quelques rares habitants du village qui viennent exceptionnellement assister au premier office du matin. Devant l'iconostase, la supérieure recommence la même opération devant chaque icône avant de déposer la coupelle sur les

\footnotetext{
${ }^{8}$ La signification de ce geste qui consiste à allumer, éteindre puis rallumer un cierge plusieurs fois à la suite peut varier selon les interlocuteurs, la plupart du temps on explique que cela correspond au nombre de personnes et de prières que l'on souhaite faire en allumant un seul cierge.

${ }^{9}$ Dans les églises byzantines, il s'agit du grand lustre suspendu à la coupole. Il symbolise la voûte céleste.

${ }^{10}$ Dans l'orthodoxie, les femmes considérées comme impures en raison des menstrues, n'ont pas l'autorisation de s'approcher de l'autel. Ainsi seules quelques femmes ménopausées peuvent passer derrière l'iconostase qui marque la séparation dans les églises orthodoxes entre le chœur de l'assemblée des fidèles et le sanctuaire. Cette règle est parfaitement connue et intériorisée par les femmes qui ne l'enfreignent jamais.

${ }^{11}$ Deux popes au village sont en charge de la vie paroissiale des grecs orthodoxes de Ma lûlâ. Ils se relaient pour célébrer les liturgies au monastère et officient ensemble le dimanche et lors des fêtes religieuses. Ils n'ont que très peu de rapport avec les religieuses. En principe, ils quittent le monastère dès que l'office est terminé. Ils célèbrent aussi les mariages, les baptêmes et les funérailles.
} 
marches du sanctuaire. Quelques instants plus tard, hajja Tedocia viendra la reprendre et derrière l'iconostase, l'encens finira de propager ses parfums.

Lorsque le pope arrive enfin, l'église est chargée des fumées d'encens. Après s'être signé et saluant rapidement d'un hochement de tête les religieuses qui ne cessent de psalmodier, il se dirige vers le sanctuaire où hajja Tedocia l'attend. Elle a déjà sorti de l'armoire le précieux habit liturgique qu'elle a elle-même cousu et brodé. En effet, avec quelques moniales du monastère et de celui de la Vierge de Saydnâyâ situé non loin, elle passe de longues aprèsmidi à confectionner les riches habits liturgiques des prêtres et prélats de l'Église. Mais pour l'heure, elle assiste le pope dans la préparation du grand encensoir à chaîne. Le pope le balance d'un geste vif tout en faisant le tour de l'autel. Puis, il fait de même à la porte du sanctuaire en direction des moniales et de l'assistance. Il accomplit ensuite les prières silencieuses et chante les psaumes. Une fois l'orthros achevé, le pope quitte rapidement le monastère.

Les moniales, quant à elles, s'attardent dans l'église et pour la deuxième fois de la matinée, elles reprennent leurs lentes déambulations pieuses. Elles embrassent à nouveau chacune des icônes de l'iconostase, se signent devant chacune d'elles et, pour certaines, effectuent une série de grandes métanies sous la coupole. Avant de sortir de l'église, elles font de nouveau trois petites métanies devant l'icône placée devant la porte. Enfin, chacune s'en va vaquer à ses tâches quotidiennes.

Ainsi quotidiennement, les moniales s'arrêtent devant chaque icône de l'église, se prosternent, embrassent et murmurent quelques suppliques aux saints. Ces gestes et ces paroles sont codifiés et sont accompagnés de petites ou de grandes métanies. Les façons, lentes et appliquées, qu'ont les moniales de toucher, d'embrasser les icônes et de leur adresser des prières, sont particulières. Les laïcs ne font jamais de même. En effet, si leurs gestes paraissent reproduire ceux des moniales, ils trahissent toutefois un certain empressement et une moindre intensité.

Les moniales du monastère de Sainte-Thècle vivent entourées d'icônes avec lesquelles, au moyen de gestes précis et de suppliques, elles établissent quotidiennement des liens étroits, « de complicitét ${ }^{12}$ ». Il se joue quelque chose d'essentiel dans le face-à-face de la moniale avec l'icône; cette relation établie dans la prière donne du sens à leur existence au monastère. 
Dans le même temps, la répétition inlassable des mêmes gestes forge, année après année, un langage corporel monastique particulier. Ainsi, ces modes de relations aux saints façonnent non seulement les corps des moniales et leurs modes de présence mais aussi leurs façons d'appréhender le monde qui les entoure.

Dans la tradition orthodoxe, l'icône a un statut très particulier. Elle n'est pas considérée comme l'œuvre d'un individu et ne doit d'ailleurs pas être signée. Les mains de l'iconographe sont censées être guidées par celles de Dieu. Afin d'atteindre l'état de grâce où ses mains accompliront l'œuvre divine, celui-ci doit pratiquer une ascèse rigoureuse en combinant jeûne et prière. Selon une telle conception, l'icône n'est ni une œuvre d'art ou une simple image, ni un ersatz ou une illustration d'un événement biblique. Dans l'orthodoxie, l'icône est chargée de la présence de l'invisible. Ainsi, elle n'est pas une forme de langage mais avant tout, un support pour la contemplation mystique ; le divin devient visible en elle.

Dès l'aurore et la première liturgie, devant chaque icône, les religieuses sont concrètement en relation avec le divin. Cette relation passe non seulement par la prière mais aussi par le corps puisqu'elles le touchent et le goûtent même par leurs baisers.

\section{UNE VIE AU MONASTERE}

Tandis qu'une à une les moniales quittent l'église, hajja Tedocia, restée seule dans le sanctuaire, range l'habit liturgique du pope dans l'armoire destinée à cet effet puis lave la pièce à grande eau. Une fois cette tâche terminée, elle rejoint tranquillement sa cellule. Éclairée par une seule fenêtre, celle-ci ne mesure pas plus d'une quinzaine de mètres carrés. Elle est composée d'un petit salon avec un canapé et un lit placé sous la fenêtre. Deux petites marches permettent d'accéder à une minuscule cuisine où elle peut manger seule ou en compagnie des autres religieuses puis une toute petite salle d'eau. Outre les nombreuses icônes disposées un peu partout sur les murs, hajja Tedocia, comme toutes les autres religieuses, a aménagé un petit autel avec icônes précieuses, cierges et encensoir à main.

Dans l'intimité de sa cellule, elle s'empresse d'allumer le petit radiateur à gaz qui diffuse rapidement une agréable chaleur. Puis, elle reprend ses prières. Pour cela, elle commence par faire brûler un morceau de charbon sur la gazinière afin d'allumer l'encens qu'elle a disposé dans le petit encensoir à main en métal travaillé. Dès que l'encens se met à fumer, elle agite l'encensoir en prononçant à voix basse quelques prières devant l'autel puis devant chacune des nombreuses icônes qui ornent de couleurs chatoyantes les murs de sa cellule. 
Cela fait plusieurs décennies que hajja Tedocia accomplit quotidiennement ces gestes. Pourtant, chaque jour, elle répète inlassablement les mêmes gestes, seule dans sa cellule ou bien en compagnie des autres religieuses ; bien plus rarement d'étrangers au monastère. Hajja Tedocia est la marraine en religion de hajja Véronique $^{13}$. Cette dernière, âgée d'une trentaine d'années, lui rend visite tous les jours. Elle est chargée de distribuer aux religieuses les dons en nourriture faits par les visiteurs et aujourd'hui, elle vient lui porter des oranges. Tandis que l'encens finit de se consumer, hajja Tedocia s'installe confortablement sur le sofa afin de faire bouillir le café à la cardamome sur une petite gazinière portable posée sur une petite table basse. Depuis la porte ouverte de la cellule, les deux religieuses regardent sans être vues les visiteurs qui commencent à affluer au monastère. Ils traversent la cour centrale pour rejoindre l'escalier qui leur permettra d'atteindre la grotte du tombeau de sainte Thècle, lieu de tous les miracles. Pelant leurs oranges et buvant leur café, elles profitent en silence du spectacle offert par les visiteurs : des familles, des hommes et des femmes seuls, des groupes d'amis mais aussi de grands groupes de pèlerins chiites venus d'Iran. Aucun de ses visiteurs ne peut se douter qu'il est observé par les moniales heureuses de les voir se rendre sur le tombeau de leur sainte.

Hormis son assistance au pope à l'église et ses travaux de couture des habits liturgiques du clergé masculin des différentes églises et monastères de la région, aucune tâche supplémentaire n'incombe à hajja Tedocia. Âgée de près de 80 ans, elle se repose. Son existence fut remplie d'événements heureux et d'autres plus dramatiques. Elle vécut en effet une existence hors du commun et fut même la plus jeune moniale de Syrie et du Liban.

À l'âge de 13 ans seulement, peu de temps après le décès de sa mère, elle quitta sa famille et sa région natale de Tripoli dans le nord du Liban, pour entrer au monastère de la Vierge à Saydnâyâ en Syrie situé non loin de Damas et du monastère de Sainte-Thècle. Si elle n'avait jusqu'alors jamais visité le monastère, elle connaissait certaines de ses moniales qui, de passage au Liban, rendaient parfois visite au foyer familial. En effet, jusque dans les années 1960, quelques moniales de Saydnâyâ voyageaient de temps à autre en Syrie et au Liban pour s'occuper des affaires courantes relatives aux nombreuses terres et propriétés waqf $(s)^{14}$ que le

\footnotetext{
${ }^{13}$ Lors de la cérémonie de prise d'habit du noviciat, chaque novice est accompagnée par celle qui est désignée comme sa marraine par la supérieure du monastère. La marraine est toujours une moniale qui a prononcé ses vœux définitifs, elle accompagne la novice dans son cheminement religieux et développe avec elle une relation de proximité et privilégiée.

${ }^{14}$ Biens de main morte.
} 
riche monastère détenait jusqu'au nord du Liban ${ }^{15}$. Elles en profitaient pour visiter certaines familles grecques orthodoxes de la région. Selon hajja Tedocia, ces visites avaient aussi pour but de repérer quelques filles aspirantes à la vie monastique. Elles organisaient également des quêtes dans les villages et les villes qu'elles traversaient. À l'occasion de ces voyages, les moniales logeaient chez les familles de notables grecs orthodoxes à Tripoli ou à Beyrouth.

Les religieuses du monastère de Saydnâyâ exerçaient ainsi une influence non négligeable parmi la communauté grecque orthodoxe aussi bien syrienne que libanaise. La mère de hajja Tédocia entretenait une relation amicale avec deux religieuses du monastère de Saydnâyâ et devant sa petite fille, elle évoquait sa vocation passée pour la vie monastique entravée par son mariage. Comme le raconte aujourd'hui hajja Tedocia, il s'agissait là pour sa mère d'une belle vie qu'elle aurait aimé mener. Lorsque sa mère mourut, la petite fille fit sien le désir de sa défunte mère et elle décida de devenir religieuse au couvent de la Vierge en Syrie. Elle confia alors son ambition à son père, qui lui opposa un refus catégorique. Néanmoins, la fillette ne se découragea pas et bien que sachant encore peu écrire, elle rédigea une lettre à l'attention de l'évêque de la région dans laquelle elle exposa sa volonté d'entrer au couvent de la Vierge à Saydnâyâ. Le prélat convainquit alors son père de la laisser partir en Syrie pour une «période d'essai » au monastère, où elle entra finalement à l'âge de 13 ans. Son père regretta immédiatement son accord et se déplaça plusieurs fois pour tenter de ramener la fillette à son foyer. Elle-même relate que, non encore rompue à la rude vie du monastère, soumise à l'isolement dans un environnement inconnu, elle s'interrogeait sur la réalité de sa propre vocation monastique et de plus, elle se sentait effrayée par la perspective de rester une vie entière au monastère. Enfin, les pleurs et le désarroi de son père étaient si difficiles à supporter qu'elle finit par se résoudre à rejoindre la maison paternelle.

Ainsi, la vocation de la petite Tedocia n'était pas encore infaillible et les doutes ne cessaient de l'assaillir jusqu'à la nuit précédant son départ qui marque pour elle la consécration de son choix difficile. Elle eut durant cette nuit-là une vision onirique de la Vierge portant Jésus dans ses bras. L’enfant dirigeait vers la petite Tedocia une grande lance enflammée. La Vierge lui parla et lui demanda de façon menaçante ce qu'elle avait l'intention de devenir hors du monastère. Hajja Tedocia raconte que non seulement elle renonça à partir du monastère mais aussi qu'à partir de ce moment-là, toute jeune qu'elle était, elle n'émit plus

\footnotetext{
${ }^{15}$ Le monastère, l'un des plus ancien de la région, a été fondé en 547. Ses propriétés s'étendent dans toute la province syrienne et vont donc au-delà des frontières nationales crées au milieu du $\mathrm{XX}^{\mathrm{e}}$ siècle.
} 
aucun doute sur le bien-fondé de sa vocation monastique. La Vierge à l'enfant menaçante qui l'empêchait de quitter son monastère lui apporta le signe de sa vocation.

Pourtant, comme son père, le patriarche de l'Église de l'époque n'était pas d'accord pour qu'elle entre si jeune dans la vie religieuse. Selon elle, c'est la supérieure du couvent qui insista auprès du patriarche pour qu'elle puisse rester au monastère. Ainsi, pour ce qui est de la communauté qu'elle dirige, la supérieure du couvent dispose d'un pouvoir véritable, supérieur même à celui du patriarche en matière d'autorité sur les religieuses. Et même si les religieuses et la supérieure sont sous l'autorité du patriarche, il est difficile pour ce dernier d'avoir un parfait contrôle sur ce qui se passe au sein des murs du lieu de culte. Le couvent est l'un des rares lieux en Syrie où les femmes possèdent de fait l'autorité. Ce pouvoir sur les hommes leur est transmis par la Vierge - patronne du monastère - qui, avec son fils dans les bras, menaçant la fuyarde d'une lance enflammée, l'interroge sur ses intentions. Ainsi, la Vierge, non seulement, confirme la décision de la supérieure et légitime la présence de la petite Tedocia au couvent, mais, de plus, l'inscrit du même coup dans un destin hors du commun qui lui fut même annoncé une deuxième fois.

Devenue donc la plus jeune des novices puis des moniales du couvent, alors qu'elle était tout juste âgée de 40 ans, elle fut envoyée en 1968 par l'évêque de Damas au monastère libanais de Nouriyyé (dans la région de Batroun) afin d'y refonder une communauté religieuse disparue depuis plusieurs siècles. Cependant, elle raconte qu'il lui était alors difficile de se décider à retourner dans le pays de son enfance. Elle avait déjà auparavant refusé de quitter le monastère pour s'occuper avec une autre religieuse d'un orphelinat à Beyrouth. Mais la veille de son départ pour le Liban, alors qu'elle ne savait que faire, elle reçut pour la deuxième fois, un signe divin : elle vit en rêve le monastère de Nouriyye qu'elle n'avait pourtant jamais visité. Ce dernier est situé dans la montagne et surplombe la mer. Elle se vit avancer vers le monastère depuis le haut d'une colline. Là elle rencontra une autre religieuse à qui elle demanda si elle avait une supérieure. Celle-ci acquiesça puis la fit entrer dans l'église où une foule nombreuse semblait l'attendre. La sœur lui donna alors un cierge de grande taille et toutes les deux se tenaient dans l'église face à la foule.

Le lendemain du songe, deux évêques grecs orthodoxes vinrent chercher hajja Tedocia et une autre religieuse pour les conduire au Liban. Ils allèrent d'abord tous ensemble dans un hôpital de Beyrouth pour rendre une visite au patriarche de l'Église alors souffrant. Puis les deux évêques insistèrent pour les accompagner visiter un orphelinat, mais hajja Tedocia 
refusa catégoriquement, persuadée de devoir se rendre au plus vite dans le monastère qu'elle avait vu en rêve. Les évêques les ont donc amenées dans le nord du Liban au-dessus de la ville de Batroun au monastère de Nouriyyé. En arrivant, hajja Tedocia reconnut immédiatement le monastère de son rêve. Hajja Tedocia en fut la supérieure jusqu'en 1998. Elle raconte ensuite qu'après le décès de la religieuse qui l'avait accompagnée à Nouriyyé depuis le monastère de la Vierge à Saydnâyâ, et après avoir pu refonder une communauté, elle décida, après en avoir obtenu l'autorisation du Patriarche, de venir dans celui de Ma ${ }^{\bar{c}}$ lûlâ en tant que simple moniale.

Ainsi, après avoir quitté le foyer paternel et être entrée au monastère de Saydnâyâ, l'existence de hajja Tedocia qui vécut toute la guerre du Liban et vint en aide à d'innombrables familles, fut riche en événements. Son arrivée au monastère de Sainte-Thècle en 1998 marquait le début d'une vie plus tranquille. Tandis que les religieuses plus jeunes sont contraintes d'effectuer de multiples corvées au monastère, les moniales plus âgées comme hajja Tedocia se dédient presque entièrement à la prière et à veiller sur le tombeau de sainte Thècle.

Vers 12 heures 30, la cloche qui annonce le repas retentit dans tout le monastère. Les religieuses laissent une à une leurs occupations afin de se retrouver dans la salle de réfectoire. Selon leurs activités, les religieuses arrivent plus ou moins rapidement. Le couvert est déjà mis et les plats sont posés sur la table lorsque hajja Tedocia arrive. La nourriture de la supérieure est, quant à elle, présentée dans des plats particuliers qu'elle ne partage pas. Une hiérarchie implicite s'exprime alors dans la manière dont les religieuses s'installent à table. La supérieure déjeune au bout de la table et donne le signal du début du repas pour les religieuses qui sont là. À sa droite et à sa gauche, sur la longue table rectangulaire se placent les religieuses en fonction de leur âge et de leur ancienneté au monastère, les plus proches de la supérieure sont les plus anciennes. Les postulantes commencent toujours le repas après tout le monde et ne s'assoient pas, elles déjeunent debout ${ }^{16}$, prêtes à apporter ou à débarrasser les plats. Hajja Tedocia vient s'asseoir près de la supérieure, à sa droite. Les religieuses occupées à préparer les plats mangent, elles, en principe, dans la cuisine. Aucune prière (bénédicité ou grâces) n'accompagne le déjeuner, qui ne dure pas plus de vingt minutes. Puis les religieuses débarrassent la table en veillant à laisser le couvert et quelques plats pour celle qui surveille la grotte du tombeau de la sainte, car très souvent à cette heure-ci, les visiteurs sont encore

\footnotetext{
${ }^{16}$ On ne retrouve cette pratique qu'au monastère.
} 
nombreux à y prier. Les religieuses organisent des rondes de garde pour ne pas fermer l'accès à la grotte pendant l'heure des repas. Dès que tous les repas sont terminés, les religieuses ferment à clé le réfectoire et la cuisine afin que les religieuses soient obligées de manger ensemble aux heures définies.

Il est maintenant 14 heures et toutes les moniales ont terminé leurs tâches. Même le commerce du monastère reste fermé pour quelque temps. Jusqu'à environ 15 heures 30, les moniales ont du temps libre et la plupart se retirent dans leurs cellules. D'autres s'installent au soleil sur les sofas disposés sur les balcons. Là, à l'abri du regard des visiteurs, par petits groupes, elles préparent café et maté sur de petites gazinières amovibles qu'elles disposent entre elles. Certaines lisent les récits hagiographiques des saintes éponymes, d'autres brodent des icônes en perle. Seul l'orphelinat est animé et les cris des pensionnaires qui jouent sur la terrasse retentissent dans la vallée.

À cette heure-ci, le visiteur est sûrement surpris de ne croiser aucune religieuse dans le monastère. Il traverse les cours sans rencontrer aucune présence, mais les religieuses, quant à elles, les observent, silencieuses derrière les moustiquaires des cellules, ou au travers des barreaux des balcons en pierre.

\section{VEILLER LA SAINTE ET PROCESSUS DE SANCTIFICATION}

Comme toutes les moniales les plus âgées, hajja Tedocia, ne se charge plus réellement des corvées du monastère. Elle s'occupe de veiller sur la grotte ou bien reste dans sa cellule. Elle se rend quelques heures par mois à l'atelier de couture. De façon générale, hajja Tedocia est moins visible que toutes les autres. Son attitude est empreinte d'une grande retenue. Après avoir pris son repas, elle va dans la grotte de la sainte où se pressent les visiteurs pour prendre la place de hajja Iman. De là, assise sur le muret, au soleil : elle dispose d'une vue panoramique sur tout ce qui se déroule dans le monastère et dans le village. Ainsi, elle observe les visiteurs qui entrent et qui au terme de leur d'ascension débouchent sur la cour principale, à côté de l'église. Elle peut suivre leur progression jusque devant la grotte où, là, ils ont la surprise de la rencontrer.

Hajja Tedocia se met alors totalement au service de sainte Thècle et des visiteurs dont on dit qu'ils sont, eux aussi, appelés par la sainte à se rendre sur son tombeau, comme les moniales furent appelées par la sainte à entrer dans son monastère. Ainsi, la religieuse chargée de veiller sur le tombeau de la sainte dit veiller la sainte elle-même et vit non plus au rythme 
du reste de la communauté monastique mais au rythme des dévots qui viennent implorer la sainte à n'importe quel moment de la journée, y compris pendant les célébrations religieuses. Les visites peuvent durer jusque tard dans la soirée et toute la nuit lors de certaines fêtes. En plus de filer du coton blanc et de donner les consignes aux visiteurs sur les façons de se comporter dans la grotte, elle a aussi pour fonction de prier pour ceux qui le souhaitent. En certaines circonstances, elle se fait aussi intermédiaire entre sainte Thècle et les visiteurs venus implorer un miracle. Elle transmet alors la bénédiction (baraka) de la sainte aux dévots.

Ces moniales anciennes au monastère ne participent donc presque pas à la vie communautaire et se consacrent entièrement à leur rapport avec sainte Thècle. L'intimité créée avec la sainte leur permet même d'avoir des discussions avec elle et de la voir souvent. Mais les récits qu'elles donnent de leurs liens avec la sainte ne décrivent pas un rapport hiérarchique ou même un rapport de type disciple à maître. Au contraire, les liens qui les unissent sont pensés comme plus complexes et renvoient à une double logique de substitution, dans laquelle les moniales se substituent à la sainte en même temps que celle-ci se substitue symboliquement à elles. C'est ainsi qu'il faut comprendre la signification d'une icône de sainte Thècle. L'icône disposée dans l'église représente sainte Thècle vêtue de l'habit monastique. Cette icône unique au monastère est en quelque sorte «anachronique » et ne renvoie à aucun élément de son hagiographie. Les moniales n'arrivèrent au monastère qu'au début $\mathrm{du} \mathrm{XX}^{\mathrm{e}}$ siècle après qu'eut été massacrée la communauté monastique masculine précédente par les soldats ottomans. Ainsi, l'icône provoque une confusion chez celui qui l'observe.

L'auréole de sainteté et la croix tenue dans la main droite du personnage font clairement référence à sainte Thècle tandis que son habit et son voile évoquent une moniale du couvent. L'ordre chronologique entre l'hagiographie de la sainte et l'histoire du monastère n'est pas respecté. Les temps du récit iconographique se superposent ou encore, sont éludés au profit d'un «hors du temps » où la sainte serait aussi moniale. Au-delà de la question de l'anachronisme, on peut se demander si l'icône représente sainte Thècle ou bien si elle représente une moniale du monastère. Est-elle celle de la moniale sainte Thècle ou celle d'une sainte moniale ?

$\mathrm{Si}$, dans la tradition orthodoxe, une icône est chargée de la présence de l'invisible et est un support pour la contemplation mystique et l'accès au divin devenu en quelque sorte visible, on peut se demander ce que dans ce cas on adore ? Cette icône montre une superposition des 
figures de la sainte et de la moniale qui pourrait typiquement être hajja Tedocia, en somme un rapport entre l'une et l'autre. Une réflexion sur cet « invisible» qui devient visible dans cette icône nous mène alors vers une analyse des rapports qui, incontestablement - l'icône nous le montre -, existent entre la sainte et les moniales qui sont aussi ses disciples les plus proches. L'icône illustre, en effet, les liens intimes unissant la sainte et les membres de la communauté monastique les plus avancées dans la vie religieuse. Ainsi, une réflexion portant, à la fois, sur l'organisation monastique générale et sur cette image religieuse spécifique permet de mettre en lumière le processus qui autorise, dans le discours des religieuses décrivant notamment les apparitions de la sainte, une substitution de la figure de la moniale à celle de la sainte. Les moniales commencent toujours leur récit en précisant que dans un premier temps elles n'étaient pas certaines de voir la sainte ou la silhouette d'une des moniales de la communauté. Seuls les chaussons d'un blanc éclatant de la sainte permettent de la reconnaître puisque les moniales elles-mêmes ne sont autorisées à porter que du noir. Cette icône de la moniale sainte Thècle ou d'une sainte moniale revêt une signification puissante, quoiqu'implicite, qui nous permet de comprendre ce qui profondément «fait» la moniale au monastère de SainteThècle.

L'identification entre sainte Thècle et les moniales se fonde sur un parallèle entre leur réclusion et leur dévouement respectifs ainsi que sur un processus singulier de sanctification progressive des moniales. Progressivement, au monastère, elles atteignent un tel niveau d'intimité avec la sainte, que dans leurs récits sur les apparitions ou les visions oniriques de la sainte, une superposition s'opère entre sa figure et celle de la moniale. Il y a une si forte identification avec la sainte que les religieuses deviennent elles-mêmes la sainte. Il n'existe plus de limite entre la sainte et les moniales, c'est même là l'un des buts d'une existence religieuse correctement accomplie au sein du monastère.

\section{LA NUIT AU MONASTERE : LE TEMPS DES APPARITIONS}

Vers 16 heures, le commerce du monastère ainsi que l'église ouvrent de nouveau leurs portes. Les religieuses qui n'ont pas d'activité l'après-midi, notamment celles qui s'occupent de la cuisine, continuent de vaquer à leurs occupations personnelles jusqu'à 17 heures 30 . Alors que le soleil décline rapidement et que les visiteurs se font moins nombreux, les religieuses se rendent dans l'église pour lire l'une après l'autre des passages de la Bible et chantent les psaumes. Elles réitèrent les gestes du matin : les petites et grandes métanies, les baisers aux icônes et à la porte de l'église. Après que le dernier visiteur quitte le monastère, 
hajja Tedocia se rend elle aussi dans l'église. Lorsque les prières sont terminées, toutes s'attardent plus encore que le matin dans l'église pour se prosterner devant les images sacrées.

Après la prière, les religieuses et les petites filles de l'orphelinat abritées dans le monastère se retrouvent sur la terrasse devant l'église qui surplombe le village et toute la vallée. Par groupe de trois ou quatre, elles font les cent pas en se tenant par les coudes, réalisant ainsi une promenade au sein même du monastère que les religieuses ne quittent jamais pour beaucoup d'entre elles. Elles parcourent ainsi toute la largeur de la terrasse pendant environ trente minutes au milieu des jeux des pensionnaires de l'orphelinat. Celles-ci disposent également d'une terrasse devant l'espace de l'orphelinat où les pensionnaires se divertissent l'après-midi alors que la partie centrale du monastère est traversée par les visiteurs. Le lieu de culte n'est pas entièrement accessible aux visiteurs et l'interdiction de l'espace desservant les cellules monastiques et la bâtisse de l'orphelinat est clairement marquée en plusieurs langues. Hajja Tedocia, se promène quelques minutes à pas lents mais rapidement elle s'assied sur les marches de l'église afin de regarder la nuit tombée sur la vallée. Elle est entourée de plusieurs petites filles visiblement très attachées à elle. Elle leur distribue des sucreries et les écoute en souriant lui raconter leurs petites histoires. Hajja Marina descend enfin fermer les portes du monastère pour la nuit et au retour, ouvre celles du réfectoire. Quelques religieuses se retrouvent afin de dîner frugalement d'olives, de pain trempé dans l'huile et de quelques légumes crus, le tout accompagné de thé. La plupart se préparent des plateaux de nourriture qu'elles montent dans leurs cellules où elles se réunissent par petits groupes.

Lorsque le soir les portes du monastère se ferment et qu'une à une les lumières des maisons de Ma ${ }^{\bar{c}}$ lûlâ s'éteignent, les religieuses commencent leurs longues veillées. Derrière les portes des cellules, elles se reçoivent entre elles et organisent de modestes dîners. Elles peuvent encore se réunir simplement autour du thé ou du café pour broder des icônes de perles qu'elles vendent ensuite aux visiteurs. La cellule de hajja Tedocia réunit plusieurs moniales et quelques jeunes filles de l'orphelinat qui dînent toutes ensemble. L'espace de l'orphelinat se trouve au cœur du monastère et les pensionnaires dont s'occupe particulièrement hajja Catherine ont d'étroites relations avec les religieuses et la mère supérieure. Si les pensionnaires disposent de leur propre réfectoire, dès qu'elles atteignent l'adolescence, elles s'organisent librement pour dîner chez l'une ou l'autre moniale qui les accueillent volontiers. Les pensionnaires circulent librement dans le monastère et aident souvent les moniales dans les tâches de nettoyage du lieu de culte par exemple. Elles participent à l'office du soir et à tous les offices de jours quand elles ne se rendent pas à 
l'école du village. Nombre d'entre elles nouent des relations particulières avec certaines moniales et l'intimité des cellules monastiques leur permet de s'isoler un peu alors que leur vie quotidienne à l'orphelinat qui compte environ une vingtaine de pensionnaires est plutôt soumise au régime de la collectivité, que ce soit au réfectoire ou dans le dortoir. Ainsi, tandis que les moniales discutent entre elles, les jeunes filles en profitent pour regarder leur feuilleton préféré à la télévision, une télévision que la plupart des religieuses ont dans leur cellule et qui leur permet d'avoir une fenêtre sur le monde extérieur malgré le fait que beaucoup ne quittent jamais le monastère. Hajja Tedocia particulièrement accueillante et bienveillante. Toutes discutent de la journée passée, des visiteurs rencontrés. Elles commentent et critiquent les faits et gestes de la supérieure ainsi que des postulantes et des jeunes novices qui lui sont alliées. Dans cette communauté cloîtrée sous l'autorité d'une supérieure, les moniales ne défient pas seulement l'ordre mondain et lignager mais aussi, l'autorité interne du monastère en utilisant la sainte comme référence ultime de l'autorité dans l'organisation monastique. Lors de ces nuits, elles aiment en effet se remémorer les miracles de la sainte, se raconter leurs rêves au sujet de la sainte qui serait venue leur parler, les rassurer, parfois les mettre en garde contre la supérieure. C'est en grande partie lors de ces nuits que la vie monastique se tisse. Forte de sa longue expérience, hajja Tedocia écoute et prodigue des conseils tout en apaisant les tensions entre moniales qui sont souvent assez intenses.

La nuit représente l'espace temporel privilégié des apparitions de sainte Thècle. Elles se déroulent toujours dans l'enceinte du monastère, et souvent à proximité des cellules situées sur ses hauteurs à proximité de la shaghûla, la grotte où se trouve son tombeau. Contrairement aux songes dans lesquels la sainte est vêtue de son habit classique, lors de ses apparitions la sainte porte l'habit monastique noir des religieuses du couvent. C'est la moniale sainte Thècle de l'icône si particulière située dans l'église et qui représente la sainte en habit monastique. Les religieuses décrivent les apparitions de la sainte ainsi vêtue en précisant que celle-ci pourrait être confondue avec l'une d'entre elles. Seuls ses chaussons d'un blanc éclatant permettent de l'identifier formellement, aucune religieuse n'étant autorisée à porter du blanc.

Les moniales évoluent au monastère en cherchant à se rapprocher le plus possible de la sainte jusqu'à établir une filiation en ligne directe avec elle afin de devenir « filles de sainte Thècle ». Puis peu à peu, la personnalité des moniales les plus anciennes «s'efface». Progressivement, elles vont à la fois s'incarner dans la sainte et laisser celle-ci s'incarner en 
elles. Après avoir fait leurs vœux perpétuels et avoir revêtu l'habit monastique entièrement noir soit environ après treize années passées au monastère comme novices ${ }^{17}-$, les moniales s'emploient à tisser des liens de plus en plus étroits avec la sainte. Elles sont sujettes à des visions, des apparitions et des songes de sainte Thècle de plus en plus récurrents qui vont de pair avec une contestation croissante de la légitimité de l'autorité de la supérieure sur la communauté. Hajja Tedocia a atteint ce stade de la vie monastique et est donc devenue un modèle pour de nombreuses religieuses. Son degré d'intimité avec la sainte est grand et les visions et les rêves sont fréquents et précis. Les moniales les plus avancées se rapprochent d'autant plus de la sainte que, dans l'espace même du monastère, elles ont la responsabilité de veiller sur son tombeau tout le jour durant, à l'exception de la mère supérieure qui ne s'y rend en prière que très rarement. Par opposition, les postulantes et les novices ne sont pas autorisées à se rendre fréquemment sur le tombeau de la sainte. Elles doivent se charger des corvées au monastère et faire leurs prières dans leurs cellules ou dans l'église.

Après, vingt années environ passées au monastère (ou dans la vie religieuse), les moniales sont autorisées à veiller la sainte, garder son tombeau et accueillir les visiteurs dans la grotte qui sont souvent nombreux et qu'il faut donc être capable de gérer. Cette activité est considérée par toutes comme un privilège. Selon la moniale Thècle ${ }^{18}$, il s'agit du meilleur endroit du monastère. Malgré les longues heures qu'elle passe assise dans la grotte, souvent dans le froid, elle dit ne ressentir aucune fatigue ou lassitude car elle est au plus près de la sainte et bénéficie ainsi de sa baraka. C'est lors de ces longues journées de veille qu'elle raconte également entretenir des conversations avec la sainte. Elle s'entretient ainsi avec elle de sujets ayant trait à la vie au monastère, lui demandant une orientation quant à diverses situations. Selon ses dires, la sainte lui parle aussi, la conseillant et l'encourageant

Tedocia est quant à elle plus discrète sur le sujet, néanmoins, elle se dit être toujours accompagnée par la sainte et exaucée par elle dans tous ses vœux.

Les veillées des moniales se finissent assez tard, aux alentours de minuit. Chacune rejoint sa cellule pour une courte nuit de quelques heures avant d'entamer une nouvelle journée de prière aux aurores.

\footnotetext{
${ }^{17}$ Aucune règle monastique ne fixe précisément la durée du noviciat mais, de façon générale, il dure entre 11 et 14 ans. Avant d'être novice, la jeune fille est postulante pendant environ 2 à 4 ans.

${ }^{18}$ Cette moniale a reçu le même nom que la sainte lors de la célébration de son noviciat.
} 


\section{CONCLUSION}

Une journée de hajja Tedocia décrit une existence monastique particulière, hors du monde et dans le monde. On comprend que la sainteté de Thècle est fondamentale et qu'elle est non seulement à la base de la fondation du monastère mais aussi à la base de la vie monastique. Son élaboration particulière définit en conséquence la construction symbolique du lieu de culte et le monachisme qui s'invente quotidiennement dans ce lieu précis.

Dans la grotte se réalise une véritable relation triadique entre la moniale, la sainte et les visiteurs. Les moniales les plus anciennes chargées de veiller la grotte s'éloignent certes de la supérieure et dans une certaine mesure des autres religieuses pour se rapprocher de la sainte mais elles se rapprochent aussi des visiteurs. Si hajja Tedocia a quitté le monde en entrant au monastère, elle le «retrouve » une trentaine d'années plus tard après avoir acquis un statut hors du commun. Toutes ces années passées au monastère, à l'abri du monde extérieur, en entretenant peu de contact avec les visiteurs et sa propre famille, ont véritablement transformé son statut non seulement au sein de la communauté monastique, mais aussi, son statut dans le monde. Elle peut désormais être en contact avec les visiteurs de la même façon que la sainte. Cette relation triadique est essentielle. Les moniales n'accomplissent pas un simple retrait du monde en entrant au monastère. Elles se préparent à être de nouveau en contact avec le monde par le biais de leurs relations avec les visiteurs. Mais cette fois, leur statut est celui du «passeur » de la baraka de la sainte. Leur retour au monde se réalise dans cette nuance. 